Comment. Math. Helv. 75 (2000) 189-199

(C) 2000 Birkhäuser Verlag, Basel

$0010-2571 / 00 / 010189-11 \$ 1.50+0.20 / 0$

Commentarii Mathematici Helvetici

\title{
Nonexistence of certain complete minimal surfaces with planar ends
}

\author{
Jaigyoung Choe* and Marc Soret
}

\begin{abstract}
In this paper we prove that two lines bounding an immersed minimal surface in a slab in $\mathbf{R}^{3}$ homeomorphic to a compact Riemann surface minus two disks and a finite number of points must be parallel. This theorem is extended to a higher dimensional minimal hypersurface. Also it is proved that if the Gauss map of a complete embedded minimal surface of finite total curvature at a planar end has order two, then the intersection of the surface with the plane asymptotic to the planar end cannot admit a one-to-one orthogonal projection onto any line in the plane.
\end{abstract}

Mathematics Subject Classification (1991). 53A10.

Keywords. Minimal surface, flux, Gauss map.

In 1956 Shiffman [S] proved a beautiful theorem on minimal surfaces lying between two horizontal planes as follows. Let $M$ be a minimal annulus in $\mathbf{R}^{3}, P_{1}, P_{2}$ horizontal planes such that $\partial M=C_{1} \cup C_{2}, C_{i} \subset P_{i}, i=1,2$. He showed that for any horizontal plane $P$ between $P_{1}$ and $P_{2}$, i) $M \cap P$ is a circle whenever $C_{1}, C_{2}$ are circles and ii) if $C_{1}, C_{2}$ are convex curves, so is $M \cap P$. For this theorem Shiffman may have been inspired by Riemann's minimal surface. That is a complete periodic minimal surface $R$ whose fundamental piece $F$, homeomorphic to an annulus and bounded by two parallel lines $l_{1}, l_{2}$, lies between two horizontal planes containing $l_{1}, l_{2}$ such that $R$ is an infinite number of stacks of the translated copies of $F$. Riemann's minimal surface has the characterizing property that it intersects every horizontal plane along a circle or a line. Indeed, Hoffman-Karcher-Rosenberg [HKR] showed that a properly embedded minimal annulus bounded by a pair of parallel lines in a slab is the fundamental piece $F$ of Riemann's minimal surface. Moreover Fang [F1] proved that a properly embedded minimal annulus bounded by a line and a circle, each lying in a horizontal plane, is also part of Riemann's example. It is not by accident that the lines in Riemann's minimal surface are all parallel: Toubiana $[\mathrm{T}]$ showed that the two lines bounding a properly embedded minimal annulus (genus 0) in a slab must be parallel.

In this paper, however, we prove that both the genus condition and the em-

Supported by KOSEF, BSRI, 98SNU-CNS Seed Money 
beddedness hypothesis in Toubiana's theorem can be dropped. To state the main theorem of this paper in a more general setting, define $S_{\theta}$ to be a screw motion in $\mathbf{R}^{3}$ followed by a horizontal translation, that is, a rotation $r_{\theta}$ about a vertical line by an angle of $\theta$ followed by a vertical translation $t_{v}$ and by a horizontal translation $t_{h}$. $S_{\theta}$ defines the quotient space $\mathbf{R}^{3} / S_{\theta}$.

Theorem 1. If $0<\theta<2 \pi$, then there exists no complete immersed minimal surface of finite total curvature in $\mathbf{R}^{3} / S_{\theta}$ with an embedded planar end whose intersection with the asymptotic horizontal plane $P \subset \mathbf{R}^{3} / S_{\theta}$ admits a one-to-one orthogonal projection onto a line in P.

Pérez-Ros obtained a similar result [PR, Theorem 4] under the assumption that the minimal surface has genus 1 . Incidentally the minimal surfaces that are proved not to exist by Toubiana, by us in the above theorem, and by Pérez-Ros would have one property in common if they existed: the order of the Gauss map at a planar end of the minimal surface would be two. In fact Hoffman and Karcher raised a question of whether the order of the Gauss map at a planar end of a complete embedded minimal surface of finite total curvature can be equal to two [HK, p.78]. We give a partial answer to this question.

Theorem 2. If the Gauss map of a complete embedded minimal surface $M \subset \mathbf{R}^{3}$ of finite total curvature at a planar end asymptotic to a plane $P$ has order two, then $M \cap P$ cannot admit a one-to-one orthogonal projection onto any line in $P$.

The key idea in our argument is to use the flux along a closed curve near the horizontal planar end. Along a loop surrounding the planar end the flux is zero. But there exist two oriented loops whose sum is homologous to the loop surrounding the planar end such that if the Gauss map at the planar end has order two, then the flux along either of the two loops is nonvertical. On Riemann's minimal surface, for example, the flux along any horizontal circle is nonvertical.

This argument works also for higher dimensional minimal submanifolds in $\mathbf{R}^{n}$. We can show the nonexistence of a minimal hypersurface in $\mathbf{R}^{n}$ which is regular at infinity and bounded by two nonparallel $(n-2)$-planes (Theorem 3 ).

\section{Flux lemma}

Let $\psi: M \rightarrow \mathbf{R}^{3}$ be a complete conformal minimal immersion of finite total curvature with embedded ends. There exist $p_{1}, \cdots, p_{l}$ in the closure $\bar{M}$ of the Riemann surface $M$ with $M=\bar{M} \sim\left\{p_{1}, \cdots, p_{l}\right\}$ such that the Gauss map $g$ : $M \rightarrow S^{2}$ extends to a holomorphic map $g: \bar{M} \rightarrow S^{2}$. Let $E_{1}, \cdots, E_{l}$ be the ends of $M$ corresponding to the punctures $p_{1}, \cdots, p_{l}$, respectively, with $E_{1}, \cdots, E_{m}$ being planar ends and $E_{m+1}, \cdots, E_{l}$ catenoidal ends. If $P$ is the horizontal plane 
asymptotic to an end $E_{i}, 1 \leq i \leq m$, and if the Gauss map has a zero or a pole of order $k$ at $p_{i}$, then $E_{i} \cap P \sim B, B$ being a large ball, consists of $2 k-2$ curves which are asymptotic to $2 k-2$ rays on $P$ making an equal angle of $\pi /(k-1)$. In particular, if $g$ has a zero (or a pole) of order 2 at $p_{i}$, then $M \cap P$ is an immersion of $\mathbf{R}^{1}$ which is asymptotically parallel to a line; here, and henceforth, $M$ denotes $\psi(M)$ unless there is confusion.

Assume that $d h$ is a holomorphic one-form on $M$ arising from the $z$-coordinate function on $M$, or precisely, from $z \circ \psi$ :

$$
z(p)=\operatorname{Re} \int^{p} d h, p \in M
$$

From the Weierstrass representation we have

$$
\psi(p)=\operatorname{Re} \int^{p}\left(\frac{1}{2}\left(-g+g^{-1}\right) d h, \frac{i}{2}\left(g+g^{-1}\right) d h, d h\right) .
$$

One knows that at an end $E$ of $M d h$ is either regular or has a simple pole depending on whether $E$ is planar or catenoidal (see Proposition $2.1[\mathrm{HK}]$ ). At a planar end where $g$ has a zero or pole of order $k, d h$ has a zero of order $k-2 \geq 0$ (Proposition $2.5[\mathrm{HK}]$ ).

Let $\Gamma(s)$ be a curve on $M$ with arclength parameter $s$ and $\nu(s)$ the unit conormal to $\Gamma(s)$ such that $\left\{\nu(s), \Gamma^{\prime}(s)\right\}$ determines the orientation of $M$. The flux of $M$ along $\Gamma$ is defined by

$$
\operatorname{Flux}(\Gamma)=\int_{\Gamma} \nu(s) d s
$$

It is well known (see [F2]) that

$$
\operatorname{Flux}(\Gamma)=\operatorname{Im} \int_{\Gamma}\left(\frac{1}{2}\left(-g+g^{-1}\right) d h, \frac{i}{2}\left(g+g^{-1}\right) d h, d h\right)
$$

Flux Lemma. Let $E$ be an embedded end of $M$ corresponding to a puncture $p \in \bar{M}$ and asymptotic to a horizontal plane $P$. Suppose that $M \cap P$ admits a one-to-one orthogonal projection onto a line $l \subset P$. If $\Gamma \subset M$ is a closed curve sufficiently close to $P$, then $\operatorname{Flux}(\Gamma)$ is nonvertical. Furthermore $\operatorname{Flux}(\Gamma)$ is orthogonal to $M \cap P$ if $M \cap P$ is a line.

Proof. Suppose $\operatorname{Flux}(\Gamma)$ is vertical. As we see in (1) and (3) Flux is conjugate to $\psi$. Hence the facts that $\operatorname{Flux}(\Gamma)$ has zero horizontal component and that $\psi$ has no real period imply

$$
\int_{\Gamma}\left(-g+g^{-1}\right) d h=0, \int_{\Gamma}\left(g+g^{-1}\right) d h=0 .
$$


Therefore

$$
\int_{\Gamma} g d h=0, \int_{\Gamma} g^{-1} d h=0
$$

Now we may assume that $g$ has a zero at $p$. Then $g d h$ is regular at $p$ and consequently it is regular along $(M \cap P) \cup\{p\}$. Since $\Gamma$ is sufficiently close to $P, g d h$ has no pole in the closure of the domain $D \subset \bar{M}$ bounded by $\Gamma$ and $(M \cap P) \cup\{p\}$. Hence

$$
\int_{\Gamma} g d h=\int_{M \cap P} g d h=0 .
$$

As for the line $l$ in relation to $g: M \subset \mathbf{R}^{3} \rightarrow \mathbf{C}$, we may assume $l$ is parallel to the imaginary axis of $\mathbf{C}$. Then Re $g$ does not change sign along $M \cap P$, which contradicts (4). Thus Flux $(\Gamma)$ must be nonvertical.

Suppose $M \cap P=l$. Taking a suitable conformal coordinate $\zeta$ in a neighborhood $N \subset \bar{M}$ of $p=\zeta(0)$, we may assume that

$$
g(\zeta)=\zeta^{2}, d h=\left(a_{0}+a_{1} \zeta+\zeta^{2} h(\zeta)\right) d \zeta, l \cap N=(-b i, b i),
$$

where $a_{0}, b$ are real, $h(\zeta)$ is analytic in $N$, and $(-b i, b i)$ is the line segment from $-b i$ to $b i$. Let $C_{\epsilon} \subset N$ be the circle of radius $\epsilon$ with center at $p$ and let $\bar{C}_{\epsilon} \subset C_{\epsilon}$ be the semicircle such that $\bar{C}_{\epsilon} \cap l=\{-\epsilon i, \epsilon i\}$ and $\bar{C}_{\epsilon} \cup l \sim(-\epsilon i, \epsilon i)$ is homologous to $\Gamma$. $g$ takes on real values along $l$ because $l$ is parallel to the imaginary axis. Hence

$$
\operatorname{Im} \int_{l \sim(-\epsilon i, \epsilon i)} \frac{i}{2}\left(g+g^{-1}\right) d h=0 .
$$

Note that $\operatorname{Flux}\left(C_{\epsilon}\right)$, the flux of the planar end $E$ along the curve $C_{\epsilon}$ surrounding the point at infinity $p$, is zero (see $[\mathrm{F} 2, \mathrm{p} .85]$ ). Thus, as above, we get

$$
\int_{C_{\epsilon}} g^{-1} d h=0
$$

Then

$$
\int_{C_{\epsilon}} g^{-1} d h=\int_{C_{\epsilon}}\left(\frac{a_{0}}{\zeta^{2}}+\frac{a_{1}}{\zeta}+h(\zeta)\right) d \zeta=0,
$$

which implies $a_{1}=0$. Hence

$$
\lim _{\epsilon \rightarrow 0} \operatorname{Im} \int_{\bar{C}_{\epsilon}} \frac{i}{2}\left(g+g^{-1}\right) d h=\lim _{\epsilon \rightarrow 0} \operatorname{Im} \frac{i}{2}\left[-\frac{a_{0}}{\zeta}\right]_{-\epsilon i}^{\epsilon i}=0 .
$$

Therefore from $(5),(6)$ we conclude that $\operatorname{Flux}(\Gamma)$ has zero $y$-component and so it is perpendicular to $l$.

Remark 1. The hypothesis of Flux Lemma that $M \cap P$ be a graph over $l$ is delicate in that there is an immersed minimal surface which has a planar end with vertical flux and whose intersection with the asymptotic plane $P$ cannot be a graph over any line in $P$ (see Section 3 ). However, we do not know whether an embedded minimal surface can have a planar end whose intersection with $P$ is not a graph. 


\section{Main theorems}

Observe that the flux of Riemann's minimal surface $M$ along a horizontal circle in $M$ is nonvertical. It is this observation that motivated us to prove Flux Lemma in the preceeding section. In this section we apply the tilting of the flux to obtain some nonexistence results for complete minimal surfaces with a planar end in $\mathbf{R}^{3}$ and in $\mathbf{R}^{n}$. Recall that $\mathbf{R}^{3} / S_{\theta}$ is the quotient space defined by $S_{\theta}$, the screw motion followed by a horizontal translation $t_{h}$.

Theorem 1. Suppose that $M \subset \mathbf{R}^{3} / S_{\theta}$ is a complete immersed minimal surface of finite total curvature with an embedded planar end $E$ which is asymptotic to a horizontal plane $P$. If $M$ intersects $P$ along a curve $\Gamma$ which admits a one-to-one orthogonal projection onto a line in $P$, then $\theta=0$. Consequently if $0<\theta<2 \pi$ then no such minimal surface exists.

Proof. Let $\widetilde{M}$ be the minimal surface in $\mathbf{R}^{3}$ associated with $M \subset \mathbf{R}^{3} / S_{\theta}$, that is, $\widetilde{M}$ is invariant under $S_{\theta}$ and $\widetilde{M} / S_{\theta}=M$. Choose $\widetilde{P}$ to be a plane in $\mathbf{R}^{3}$ associated with $P \subset \mathbf{R}^{3} / S_{\theta}$ and $\bar{P}$ a plane parallel to $\widetilde{P}$. If $\bar{P}$ is close enough to $\widetilde{P}$, then by Flux Lemma $v=\operatorname{Flux}(\widetilde{M} \cap \bar{P})$ is nonvertical. Assume $v$ is computed with the upward conormals. Let $M^{\prime}$ be the subset of $\widetilde{M}$ lying between $\bar{P}$ and $S_{\theta}(\bar{P})$. The boundary of $M^{\prime}$ consists of $\widetilde{M} \cap \bar{P}, \widetilde{M} \cap S_{\theta}(\bar{P})$, and the punctures corresponding to the planar ends. Since the flux of $\widetilde{M}$ along a curve surrounding the point at infinity of a planar end is zero, the downward flux of $\widetilde{M}$ along $\widetilde{M} \cap S_{\theta}(\bar{P})$ equals $-v$. But the invariance of $\widetilde{M}$ under $S_{\theta}$ also implies that the downward flux of $\widetilde{M}$ along $\widetilde{M} \cap S_{\theta}(\bar{P})$ equals $-S_{\theta}(v)$. Therefore $-v=-S_{\theta}(v)$, and this holds only when $\theta=0$ since $v$ is nonvertical.

Theorem 1 gives an extension of Toubiana's result as follows.

Corollary. Let $R$ be a compact Riemann surface containing two disks $D_{1}, D_{2}$ and points $p_{1}, \ldots, p_{n}$, and let $S \subset \mathbf{R}^{3}$ be a slab. If $\psi: R \sim\left(D_{1} \cup D_{2} \cup\left\{p_{1}, \ldots, p_{n}\right\}\right) \rightarrow S$ is a minimal immersion of finite total curvature such that $\psi\left(\partial D_{1}\right)$ and $\psi\left(\partial D_{2}\right)$ are straight lines in $\partial S$, then the lines are parallel.

Proof. Rotating $M$ around boundary lines by $180^{\circ}$, we get a minimal surface in $\mathbf{R}^{3} / S_{\theta}$ such that $\theta / 2$ is the angle the two boundary lines of $M$ make. By Theorem $1 \theta=0$, which means that the two lines are parallel.

Question. Is there a minimal surface which is invariant under $S_{\theta}$ for a nonzero $\theta$ and a nonzero horizontal vector $h$ ? Riemann's minimal surface is invariant under $S_{\theta}$ for $\theta=0$ and nonzero $h$. The helicoid is invariant under $S_{\theta}$ for a nonzero $\theta$ and $h=0$. 
The following theorem deals with a situation relevant to Hoffman and Karcher's question.

Theorem 2. Let $M \subset \mathbf{R}^{3}$ be a complete embedded minimal surface of finite total curvature with a planar end $E$ asymptotic to a plane P. If the Gauss map has order two at $E$, then $M \cap P$ cannot admit a one-to-one orthogonal projection onto any line in $P$.

Proof. Suppose $E \cap P$ admits a one-to-one orthogonal projection onto a line in $P$. Take a Jordan curve $c_{0}$ which is sufficiently close to $P$. $c_{0}$ divides $M$ into two components, with $M^{\prime}$ one of them. Let $c_{1}, \cdots, c_{m} \subset M^{\prime}$ be mutually disjoint Jordan curves winding each end of $M^{\prime}$ such that $\sum_{i=0}^{m} c_{i}=0$ with respect to appropriate orientations for $c_{0}, c_{1}, \cdots, c_{m}$. Then we have

$$
\sum_{i=0}^{m} \operatorname{Flux}\left(c_{i}\right)=0
$$

Flux $\left(c_{i}\right), i=1, \cdots, m$, is either vertical or zero depending on whether the corresponding end is catenoidal or planar. But Flux Lemma says Flux $\left(c_{0}\right)$ is nonvertical. Then (7) cannot hold. This completes the proof.

Finally let us extend the above corollary to the case of minimal hypersurfaces in $\mathbf{R}^{n}$. This extension will apply only to hypersurfaces which are well behaved at infinity. These hypersurfaces, in a sense, are higher-dimensional versions of two-dimensional minimal surfaces of finite total curvature with embedded ends. $\mathrm{R}$. Schoen defined in [Sc] that a complete immersed hypersurface $M^{n-1} \subset \mathbf{R}^{n}$ is regular at infinity if there is a compact subset $K \subset M$ such that $M \sim K$ is a union of $E_{1}, \cdots, E_{m}$ where each $E_{i}$, called an end of $M$, is a graph of bounded slope over the exterior of a bounded region in a hyperplane $P_{i}$. He showed that if $x_{1}, \cdots, x_{n-1}$ are coordinates in $P_{i}$, then $E_{i}$ is the graph of a function $u_{i}$ with the following asymptotic behavior for $|x|$ large and $n \geq 4$ :

$$
u_{i}(x)=b+a|x|^{3-n}+\sum_{j=1}^{n-1} c_{j} x_{j}|x|^{1-n}+O\left(|x|^{1-n}\right)
$$

for constants $a, b, c_{j}$ depending on $i$. The expression $O\left(|x|^{1-n}\right)$ in the above equation is used to indicate a term which is bounded in absolute value by a constant times $|x|^{1-n}$ for $|x|$ large.

For the following theorem define a slab $\Pi=\left\{\left(x_{1}, \cdots, x_{n}\right) \in \mathbf{R}^{n}: a \leq x_{n} \leq b\right\}$ for some constants $a<b$. And for the Schwarz reflection in higher dimensions as stated in the following theorem, the readers are referred to [GT].

Theorem 3. Assume that $M^{n-1} \subset \mathbf{R}^{n}, n \geq 4$, is an immersed minimal hypersurface in a horizontal slab bounded by two $(n-2)$-dimensional planes $Q_{1}, Q_{2}$. 
Extending $M$ across the boundary planes by repeated $180^{\circ}$ rotations, one obtains a complete minimal hypersurface $\bar{M}$; suppose $\widetilde{M}$ is regular at infinity. Then $Q_{1}$ and $Q_{2}$ are parallel.

Proof. The regularity of $\widetilde{M}$ implies that $M$ is diffeomorphic under a diffeomorphism $\phi$ to a compact manifold $\bar{M}$ with boundary punctured at $p_{1}, \cdots, p_{m+2} \in \bar{M}$ with $p_{m+1}, p_{m+2} \in \partial \bar{M}$. So $p_{1}, \cdots, p_{m}$ correspond to the ends $E_{1}, \cdots, E_{m}$ of $M$, respectively, and $\partial \bar{M} \sim\left\{p_{m+1}, p_{m+2}\right\}=\phi\left(Q_{1} \cup Q_{2}\right)$. Define closed $(n-2)-$ dimensional surfaces $S_{i}$ and $S_{m+j} \subset M$ winding around the end $E_{i}, i=1, \cdots, m$, and the half end $E_{m+j}, j=1,2$, respectively, as follows. For a small neighborhood $V_{i} \subset \bar{M}$ of $p_{i}$, let $S_{i}=\phi^{-1}\left(\partial V_{i}\right), i=1, \cdots, m$, and $S_{m+j}=\left(Q_{j} \cup \phi^{-1}\left(\partial V_{m+j}\right)\right) \sim$ $\left(Q_{j} \cap \phi^{-1}\left(V_{m+j}\right)\right), j=1,2$.

One can define the flux of $M$ along a hypersurface $S$ of $M$ by extending the definition (2):

$$
\operatorname{Flux}(S)=\int_{S} \nu
$$

where $\nu$ is a unit conormal to $S$. Obviously $\operatorname{Flux}(S)$ changes sign as one changes the orientation of $S$. Now one can easily see that

$$
\sum_{i=1}^{m+2} \operatorname{Flux}\left(S_{i}\right)=0 .
$$

Claim 1. $\operatorname{Flux}\left(S_{i}\right)$ is vertical for $i=1, \cdots, m$.

Suppose $E_{i}$ is the graph of $u_{i}$ over the exterior of a ball $B_{R}$ of radius $R$ in a hyperplane $P_{i}$. By (8) $E_{i}$ is either asymptotic to an end of a higher-dimensional catenoid $(a \neq 0)$ or planar $(a=0)$. If $a \neq 0$, then $\operatorname{Flux}\left(S_{i}\right)$ is the same as that of the catenoid along its waist, which is vertical. If $a=0$, then

$$
\left|\nabla u_{i}(x)\right|=O\left(|x|^{1-n}\right) .
$$

Let $S_{i}^{r} \subset E_{i}$ be the set whose projection onto $P_{i}$ is $\partial B_{r}, r>R$. Then $S_{i}^{r}$ is homologous to $S_{i}$ and hence for any $r>R$

$$
\operatorname{Flux}\left(S_{i}\right)=\operatorname{Flux}\left(S_{i}^{r}\right) .
$$

Let $\nu, \bar{\nu}$ be the unit conormals to $S_{i}^{r}$ in $E_{i}$ and to $\partial B_{r}$ in $P_{i}$, respectively, and Proj: $E_{i} \rightarrow P_{i}$ the projection map. Then from (10) we see that

$$
|\nu(y)-\bar{\nu}(\operatorname{Proj}(y))|=O\left(|y|^{1-n}\right), y \in S_{i} .
$$

Hence

$\operatorname{Flux}\left(S_{i}^{r}\right)=\int_{S_{i}^{r}} \bar{\nu}+O\left(r^{1-n}\right) \cdot \operatorname{Volume}\left(S_{i}^{r}\right)=\int_{\partial B_{r}} \bar{\nu}+O\left(r^{1-n}\right) \cdot \operatorname{Volume}\left(\partial B_{r}\right)$ 
Since $\int_{\partial B_{r}} \bar{\nu}=0$ for any $r$, the claim follows from (11), (12).

Claim 2. Flux $\left(S_{m+j}\right), j=1,2$, has a nonzero horizontal component perpendicular to $Q_{j}$.

Let $E_{m+j}$ be the half-end corresponding to $p_{m+j}$. Note that $a$ in (8) should vanish on $E_{m+j}$ since the $(n-2)$-dimensional plane $Q_{j}$ bounds $M$ on $E_{m+j}$. Suppose $E_{m+j}$ is the graph of $u_{m+j}$ over the exterior of a half-ball $H_{R}$ of radius $R$ in the half-plane $\left\{\left(x_{1}, \cdots, x_{n-1}, c\right): x_{n-1} \geq 0\right\} \subset P_{m+j}$. Let $S_{m+j}^{r} \subset E_{m+j} \cup Q_{j}$ be the set whose projection onto $P_{m+j}$ is $\partial H_{r}$, and $\nu, \bar{\nu}$ the unit conormals to $S_{m+j}^{r}$ in $M$ and to $\partial H_{r}$ in $P_{m+j}$, respectively. Denote the half-sphere $\partial H_{r} \sim Q_{j}$ by $J_{r}$ and set $D_{r}=\partial H_{r} \cap Q_{j}$. Then

$$
\begin{aligned}
\operatorname{Flux}\left(S_{m+j}\right)=\operatorname{Flux}\left(S_{m+j}^{r}\right) & =\int_{D_{r}} \nu+\int_{S_{m+j}^{r} \sim D_{r}} \bar{\nu}+O\left(r^{1-n}\right) \cdot \operatorname{Volume}\left(S_{m+j}^{r} \sim D_{r}\right) \\
& =\int_{D_{r}} \nu+\int_{J_{r}} \bar{\nu}+O\left(r^{1-n}\right) \cdot \operatorname{Volume}\left(J_{r}\right) .
\end{aligned}
$$

Observe that for any $r$

$$
\int_{J_{r}} \bar{\nu}=-\int_{D_{r}} \bar{\nu}
$$

Thus, letting $r \rightarrow \infty$, we get

$$
\operatorname{Flux}\left(S_{m+j}\right)=\int_{Q_{j}}(\nu-\bar{\nu}) .
$$

Since $M$ lies in a slab $\Pi$ with $Q_{j} \subset \partial M \subset \partial \Pi$ one checks that $\nu$ has a nonzero vertical component. Therefore $\nu-\bar{\nu}$ has a nonzero horizontal component. This horizontal component must be perpendicular to $Q_{j}$ because $\nu$ and $\bar{\nu}$ are. So the claim follows. Now one derives from (9) and Claim 1 that

$$
\text { the horizontal component of }\left[\operatorname{Flux}\left(S_{m+1}\right)+\operatorname{Flux}\left(S_{m+2}\right)\right]=0 .
$$

But if $Q_{1}$ is not parallel to $Q_{2}$, then Claim 2 contradicts (13). This completes the proof of Theorem 3.

\section{A counterexample}

In this section we will see an example that shows the delicacy of Flux Lemma. A minimal surface with vertical flux and with a horizontal planar end of order one (i.e. whose Gauss map has a zero or a pole of order two at the end) may have, at 
the level of the asymptotic plane, a level curve which is not a graph with respect to any line contained in the asymptotic plane (see Figure). As the example below will show, this can be the case for a minimal annulus immersed in a horizontal slab, transversal to any horizontal plane, bounded by a convex curve in the bottom plane and by a closed immersed curve in the top plane. Note that it is unknown whether such an example exists if the minimal annulus is embeddded and bounded by two closed curves. Note however that both boundary curves cannot be convex (see Pérez-Ros [PR]).

More precisely let us construct a minimal immersion $\psi$ of the doubly punctured complex disc around 0 , of radius $1+\alpha>1$

$$
\psi: D(0,1+\alpha) \sim\{0,1\} \subset \mathbf{C} \longrightarrow M \subset \mathbf{R}^{3}
$$

Points $\{0,1\}$ will correspond, respectively, to a catenoid-type end and to a planar end of order one. We define $\psi$ by its Weierstrass data as follows.

(A) Choose $\psi$ such that circles around 0 in $D(0,1+\alpha)$ parametrize the level curves of $M$. This forces

$$
d h=\frac{d z}{z}
$$

Thus $X_{3}=\log |z|$ and the planar end will be at level zero and the top curve at level $\log (1+\alpha)$.

(B) Choose the Gauss map to point upwards at 0 and downwards at 1 . But 1 becomes a planar end of order one; thus the Weierstrass data $g$ has a zero of order 2 at 1 and a pole of order one at 0 . Moreover $M$ is transversal to any horizontal plane, which gives

$$
g(z)=\frac{(z-1)^{2}}{z} R(z)
$$

where $R$ is a holomorphic function on $D(0,1+\alpha)$ with no zeroes.

It is then easy to check that $\psi$ is an immersion: the metric $d s=|z|^{-1}(|g|+$ $\left.|g|^{-1}\right)$ does not vanish on $D(0,1+\alpha) \sim\{0,1\}$. To be well defined, $\psi$ should have no real period for any Jordan curve in $D(0,1+\alpha) \sim\{0,1\}$. Also $\psi$ is assumed to have vertical flux along the circles $\Gamma$ around 0 . Hence we get

$$
\int_{\Gamma}\left(\frac{1}{2}\left(-g+g^{-1}\right) d h, \frac{i}{2}\left(g+g^{-1}\right) d h\right)=(0,0)
$$

Take two generators of $\pi_{1}(D(0,1+\alpha) \sim\{0,1\})$ given by two small curves respectively around 0 and 1 . A direct computation yields $\left((z-1)^{2} R(z)\right)^{\prime}(0)=0$ and $(1 / R(z))^{\prime}(1)=0 . R$ does not vanish, hence

$$
R^{\prime}(0)=2 R(0), R^{\prime}(1)=0 .
$$


Finally the construction is reduced to finding a holomorphic map $R$ on $D(0,1+$ $\alpha) \sim\{0,1\}$ such that

$$
\begin{aligned}
R^{\prime}(0) & =2 R(0)(\mathrm{i}) \\
R^{\prime}(1) & =0 \text { (ii) } \\
R(z) & \neq 0, \quad z \in D(0,1+\alpha) .(\mathrm{iii})
\end{aligned}
$$

Suppose

$$
R(z)=(z+p)^{n}-\epsilon z^{N}, \quad p \in \mathbf{R}, n, N \in \mathbf{N} .
$$

Then (i) gives $p=n / 2$ and (ii) gives $\epsilon=n\left(1+\frac{n}{2}\right)^{n-1} N^{-1}$. Furthermore (iii) holds if

$$
\left(\frac{n}{2}-1-\alpha\right)^{n}-n\left(1+\frac{n}{2}\right)^{n-1} N^{-1}(1+\alpha)^{N}>0 .
$$

A fortiori, for $\alpha=0$,

$$
N>\frac{n\left(1+\frac{n}{2}\right)^{n-1}}{\left(\frac{n}{2}-1\right)^{n}}
$$

This latter inequality gives the former inequality for $\alpha$ small enough. In particular, if $n=3$, then $N>150$. Take $N=151$; then

$$
R(z)=\left(z+\frac{3}{2}\right)^{3}-\frac{75}{604} z^{151} .
$$

If we remove a small disk around 0 , we obtain the desired minimal annulus.

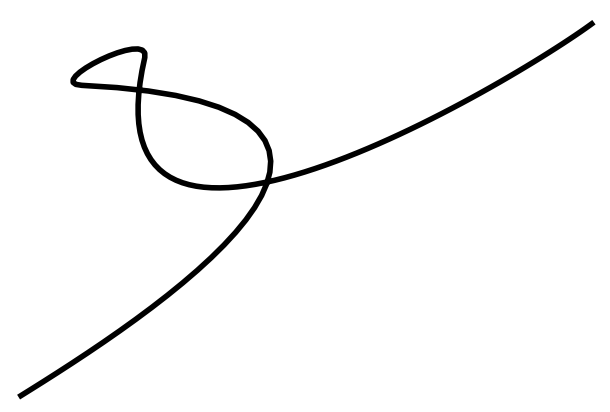

Remark 2. A sketch of the level curve $M \cap P$ at the level of the asymptotic plane $P$ in the above counterexample is drawn in the figure above. It is interesting to note that F. Lopez and F. Wei [LW] recently gave an example of a minimal annulus in a slab bounded by two figure-eight-shaped curves with a planar end whose level curve is a line. Furthermore this surface is transversal to horizontal planes and has vertical flux. However this example is an immersion of an annulus with two punctures. Thus there are indeed two planar ends at the same level with the same level curves. 


\section{References}

[F1] Y. Fang, On minimal annuli in a slab, Comment. Math. Helv. 69 (1994), 417-430.

[F2] Y. Fang, Lectures on minimal surfaces in $\mathbf{R}^{3}$, Proc. Centre for Mathematics and its Applications, Australian Nat. Univ. 35 (1996).

[GT] D. Gilbarg and N. S. Trudinger, Elliptic partial differential equations of second order, 2nd ed., Grundlehren der mathematischen Wissenschaften 224, Springer-Verlag, Berlin 1983.

[HK] D. Hoffman and H. Karcher, Complete embedded minimal surfaces of finite total curvature, Geometry V, Encyclopaedia Math. Sci. Vol. 90, Springer-Verlag 1997, pp. 5-93.

[HKR] D. A. Hoffman, H. Karcher and H. Rosenberg, Embedded minimal annuli in $\mathbf{R}^{3}$ bounded by a pair of straight lines, Comment. Math. Helv. 66 (1991), 599-617.

[LW] F. J. Lopez and F. Wei, Properly immersed minimal discs bounded by straight lines, Preprint.

[PR] J. Pérez and A. Ros, Some uniqueness and nonexistence theorems for embedded minimal surfaces, Math. Ann. 295 (1993), 513-525.

[Sc] R. Schoen, Uniqueness, symmetry, and embeddedness of minimal surfaces, J. Diff. Geom. 18 (1983), 791-809.

[S] M. Shiffman, On surfaces of stationary area bounded by two circles, or convex curves, in parallel planes, Ann. of Math. 63 (1956), 77-90.

[T] E. Toubiana, On the minimal surfaces of Riemann, Comment. Math. Helv. 67 (1992), 546-570.

Jaigyoung Choe

Department of Mathematics

Seoul National University

and Korea Institute for Advanced Study

Seoul, 151-742

Korea

e-mail: choe@math.snu.ac.kr
Marc Soret

Département de Mathématiques

Université F. Rabelais

37200 Tours

France

e-mail: soretm@univ-tours.fr

(Received: November 26, 1998) 